OPEN ACCESS

Edited by:

Ping Wu,

Fudan University, China

Reviewed by:

Chuantao Zuo,

Fudan University, China

Yong Ji,

Capital Medical University, China

*Correspondence:

Liping Fu

flp39@163.com

Ruimin Wang

wrm@yeah.net

Received: 13 August 2021 Accepted: 22 September 2021

Published: 13 October 2021

Citation:

Fu L, Zhou Z, Liu L, Zhang J,

Xie $H$, Zhang $X$, Zhu $M$ and Wang $R$

(2021) Functional Abnormality

Associated With Tau Deposition

in Alzheimer's Disease - A Hybrid

Positron Emission Tomography/MRl

Study.

Front. Aging Neurosci. 13:758053

doi: 10.3389/fnagi.2021.758053

\section{Functional Abnormality Associated With Tau Deposition in Alzheimer's Disease - A Hybrid Positron Emission Tomography/MRI Study}

\author{
Liping Fu' ${ }^{1 *}$, Zhi Zhou ${ }^{2}$, Linwen Liü, Jinming Zhang ${ }^{4}$, Hengge Xie ${ }^{5}$, Xiaojun Zhang ${ }^{2}$, \\ Mingwei Zhu ${ }^{5}$ and Ruimin Wang ${ }^{4 *}$
}

\begin{abstract}
${ }^{1}$ Department of Nuclear Medicine, China-Japan Friendship Hospital, Beijing, China, ${ }^{2}$ Department of Neurology, China-Japan Friendship Hospital, Beijing, China, ${ }^{3}$ Medical Research Center, Peking Union Medical College Hospital, Chinese Academy of Medical Sciences and Peking Union Medical College, Beijing, China, ${ }^{4}$ Department of Nuclear Medicine, The First Medical Center, Chinese PLA General Hospital, Beijing, China, ${ }^{5}$ Department of Neurology, The Second Medical Center, Chinese PLA General Hospital, Beijing, China
\end{abstract}

Objective: To investigate the characteristics of tau deposition and its impact on functional connectivity (FC) in Alzheimer's disease (AD).

Methods: Hybrid PET/MRI scans with $\left[{ }^{18} \mathrm{~F}\right.$-THK5317 and neuropsychological assessments were undertaken in 26 participants with $A D$ and 19 healthy controls $(\mathrm{HC})$. The standardized uptake value ratio (SUVR) of $\left.{ }^{18} \mathrm{~F}\right]-\mathrm{THK} 5317$ PET imaging was compared between the AD and HC groups. Significant clusters that revealed higher tau deposition in the $\mathrm{AD}$ group compared to the $\mathrm{HC}$ group were selected as regions of interest (ROI) for FC analysis. We evaluated the difference in the FC between the two groups for each $\mathrm{ROI}$ pair. The clinical and radiological characteristics were compared between the $A D$ patients with negative $F C$ and $A D$ patients with positive $F C$ for exploratory analysis.

Results: The bilateral inferior lateral temporal lobe, dorsal prefrontal cortex, precuneus, posterior cingulate cortex, hippocampus, and occipital lobe showed significantly higher $\left[{ }^{18} \mathrm{~F}\right]-\mathrm{THK} 5317$ accumulation in $\mathrm{AD}$ patients. Decreased FC in regions with higher SUVR was observed in AD patients, and the FC strength was negatively correlated with regional SUVR. Patients with a positive FC exhibited older ages, better cognitive performances, and a lower SUVR than patients with a negative FC.

Conclusions: An impact of tau deposition was observed on FC at the individual level in $A D$ patients. Our findings suggested that the combination of tau-PET and rs-fMRI might help predict AD progression.

Keywords: Alzheimer's disease, PET/MRI, tau, fMRI, functional connectivity

\section{INTRODUCTION}

Abnormal deposition of amyloid- $\beta(A \beta)$ and tau is the hallmark of pathology in Alzheimer's disease $(\mathrm{AD})$. According to the pathological classification model, the accumulation of $\mathrm{A} \beta$ plaque (A) deposition occurs first, followed by phosphorylated tau (T) deposition and downstream neurodegenerative (N) events (Jack et al., 2018). 
Tau protein plays an important role in promoting the stability of tubulin assemblies and maintaining the microarchitecture of neurons (Kametani and Hasegawa, 2018). Recent advances in molecular neuroimaging, including positron emission tomography (PET), have enabled the identification and quantification of pathological proteins in vivo. PET with tracers, including $\left[{ }^{18} \mathrm{~F}\right]-$ THK5317 and $\left[{ }^{18} \mathrm{~F}\right]-\mathrm{AV} 1451$, is a wellestablished neuroimaging technique for measuring regional tau burden. Previous studies with tau-PET demonstrated that the spatial pattern of tau deposition was similar to the key features of the Braak histopathological stages illustrated by autopsy-based assessments (Schwarz et al., 2016). Compared with $\mathrm{A} \beta$, the spread of tau pathology shows a strong relationship with the progression of AD (Kametani and Hasegawa, 2018).

The deposition of pathological proteins contributes to the cascade of functional and morphological changes in the brain. There are extensive reports indicating that tau causes direct toxic effects on neuronal activity and synaptic plasticity in $\mathrm{AD}$, leading to disruption of functional connectivity (FC), which assesses the correlation between spontaneous activity fluctuations in remote brain regions (Busche et al., 2019). The combination of tau-PET and multi-modal MRI facilitates in vivo investigation. Recently, hybrid PET/MRI has become available in clinical practice, which provides the opportunity to combine PET and MRI in a single imaging session. Neuroimaging studies using resting state functional MRI (rs-fMRI) and tau-PET support the idea that tau disrupts $\mathrm{FC}$ in $\mathrm{AD}$ by showing that tau accumulation weakens FC.

Previous work primarily focused on FC disruption in known brain functional networks. Based on these observations, recent studies illustrated that highly connected nodes, which also are called hubs, displayed more tau pathology, and increases in tau burden were associated with decreases in FC at these same nodes (Cope et al., 2018; Yokoi et al., 2018; Franzmeier et al., 2019, 2020). These results provide evidence for the "network degeneration hypothesis," in which the spreading of pathological tau propagates trans-neuronally in a prion-like manner (Kametani and Hasegawa, 2018). However, these studies examined the alterations in FC and tau deposition separately. Therefore, the direct interaction of pathological tau deposition and regional FC and the influence on cognitive impairment remains unclear. In this study, using hybrid PET/MRI with $\left[{ }^{18} \mathrm{~F}\right]-$ THK5317, we investigated the characteristics of tau deposition and its impact on FC in AD patients at the individual level.

\section{MATERIALS AND METHODS}

\section{Participants}

Twenty-six AD and $19 \mathrm{HC}$ subjects were recruited at the Chinese PLA General Hospital. The clinical diagnosis of AD was made based on the criteria for dementia cited in the International Classification of Diseases, 10th Revision (ICD-10) and the criteria for probable AD from the National Institute of Neurological and Communicative Disorders and the Stroke/Alzheimer Disease and Related Disorders Association (NINCDS-ADRDA) (McKhann et al., 2011). All participants were clinically assessed using the Clinical Dementia Rating (CDR) (Morris, 1993), which categorized the participants as $\mathrm{HCs}(\mathrm{CDR}=0)$ or patients in the early stages of $\mathrm{AD}(\mathrm{CDR}>0.5)$. The inclusion criteria for $\mathrm{AD}$ included: (1) significant episodic memory problems reported by the patient, a relative, or caregiver; (2) impaired performance on a general cognition test [Mini-Mental State Examination (MMSE) score < 24] and in activities of daily living (ADL); (3) medial temporal lobe atrophy as assessed with the visual atrophy rating scale (Scheltens et al., 1992). Also, AD patients exhibited positive results obtained with an amyloid PET $\left[\left({ }^{11} \mathrm{C}\right)\right.$ PIB] scan. Participants were excluded if they presented one of the following criteria: (1) structural abnormalities that could result in dementia, including cortical infarction, tumors, or a subdural hematoma; (2) concurrent illnesses other than dementia that interfered with cognitive function at the time of the MRI examination; (3) metabolic conditions such as hypothyroidism, and vitamin B12 or folic acid deficiencies. The HCs had no history of neurological or psychiatric disorders, sensorimotor impairment, or cognitive complaints, no abnormal anatomical findings based on conventional brain MRI, and no evidence of cognitive deficits on neuropsychological tests. All participants exhibited right-hand dominance. Written informed consent was obtained from each participant, and this study was approved by the institutional review board of the Chinese PLA General Hospital. We conducted this study in compliance with the principles of the Declaration of Helsinki.

\section{Positron Emission Tomography/MRI Scans}

Forty minutes after the injection of approximately $370 \mathrm{MBq}$ (4.44-5.55 MBq $/ \mathrm{kg}$ ) of $\left[{ }^{18} \mathrm{~F}\right]-\mathrm{THK} 5317$, each participant was positioned in a hybrid PET/MRI system (Biograph mMR, Siemens Healthineers, Erlangen, Germany) that consisted of a whole-body PET and a 3.0-T MRI. This hybrid scanner enables the acquisition of 127 transaxial planes over a $25.8 \mathrm{~cm}$ axial field of view, which allows the entire brain to be imaged in a one-bed position. The $\left[{ }^{18} \mathrm{~F}\right]-\mathrm{THK} 5317 \mathrm{PET}$ scan started $40 \mathrm{~min}$ after the tracer was injected and lasted for $20 \mathrm{~min}$. It was carried out simultaneously with the MRI scan, which included attenuation correction acquisition (ultra-short echo time (UTE) sequence, $\mathrm{TE} 1 / \mathrm{TE} 2=0.07 / 2.46 \mathrm{~ms}$, TR $=11.94 \mathrm{~ms}$, flip angle $10^{\circ}, 192$ slices, matrix size: $192 \times 192 \times 192$, FOV $=300 \mathrm{~mm} \times 300 \mathrm{~mm}$, voxel size $1.6 \mathrm{~mm} \times 1.6 \mathrm{~mm} \times 1.6 \mathrm{~mm}$, acquisition time 1:40 $\mathrm{min} / \mathrm{bed}$ position), high-resolution sagittal 3D T1-weighted magnetization-prepared rapid gradient echo (MPRAGE) acquisition ( $\mathrm{TR}=1,900 \mathrm{~ms}, \mathrm{TE}=2.44 \mathrm{~ms}$, inversion time $=900 \mathrm{~ms}$, slice thickness $=1 \mathrm{~mm}$, matrix $=256 \times 256, \mathrm{FOV}=250 \mathrm{~mm} \times 250 \mathrm{~mm}$, voxel size $1.0 \mathrm{~mm} \times 1.0 \mathrm{~mm} \times 1.0 \mathrm{~mm}$, acquisition time $8: 54 \mathrm{~min} /$ bed position), a transverse T2-weighted turbo spin echo acquisition $\left(\mathrm{TR}=4,500 \mathrm{~ms}, \mathrm{TE}=85 \mathrm{~ms}\right.$, flip angle $150^{\circ}, 25$ slices, slice thickness $4 \mathrm{~mm}, \mathrm{FOV}=220 \mathrm{~mm} \times 220 \mathrm{~mm}$, voxel size $0.7 \mathrm{~mm} \times 0.7 \mathrm{~mm} \times 4.0 \mathrm{~mm}$, acquisition time $2: 32 \mathrm{~min} / \mathrm{bed}$ position) and single shot gradient echo-planner imaging with blood oxygenation level dependence (BOLD, TR $=2,000 \mathrm{~ms}$, $\mathrm{TE}=30 \mathrm{~ms}$, slice thickness $=3.5 \mathrm{~mm}$, slice $=43$, Matrix $=64 \times 64$, 
$\mathrm{FOV}=224 \mathrm{~mm} \times 224 \mathrm{~mm}$, acquisition time $8: 00 \mathrm{~min} / \mathrm{bed}$ position). The $20 \mathrm{~min}$ (taken at $40-60 \mathrm{~min}$ ) of PET data were converted into standardized uptake value (SUV) images for further analysis using an ordered subset expectation maximization algorithm with settings of iterate $=3$, subset $=21$, matrix $=336 \times 336$, and a Gaussian filter of $4 \mathrm{~mm}$ in full-width half-maximum (FWHM). All subjects underwent THK5317-PET and 3D-T1WI MRI scanning; $22 \mathrm{AD}$ and $12 \mathrm{HC}$ subjects had resting-state BOLD data that were acquired.

All participants underwent a 20 -min $\left[{ }^{11} \mathrm{C}\right]-\mathrm{PIB}$ PET/MRI static scan, which was performed $40 \mathrm{~min}$ after injection (40$60 \mathrm{~min}$ ) of $4.5 \mathrm{MBq} / \mathrm{kg}$ (McNamee et al., 2009). The $\left[{ }^{11} \mathrm{C}\right]-$ PIB was synthesized from its corresponding precursors as described previously (Philippe et al., 2011), with a radiochemical purity of more than $95 \%$ and specific activity of $50 \mathrm{GBq} / \mathrm{moL}$ $(1.48 \mathrm{Ci} / \mathrm{moL})$. The $\left[{ }^{11} \mathrm{C}\right]-\mathrm{PIB} \mathrm{PET} / \mathrm{MRI}$ scan protocol was the same as the $\left[{ }^{18} \mathrm{~F}\right]-\mathrm{THK} 5317 \mathrm{PET} / \mathrm{MRI}$ scan.

\section{Radiosynthesis of $\left[{ }^{18} \mathrm{~F}\right]-$ THK5317}

$\left[{ }^{18} \mathrm{~F}\right]$-THK5317 was prepared using ${ }^{18} \mathrm{~F}$ - nucleophilic substitution of the tosylate in the precursor in the presence of K2CO3 and Kryptofix-222 using the automatic synthesis module (PET-MF-2V-IT-1, Beijing, China). After radiofluorination, the tetrahydropyranyl (THP) protecting group was removed using $\mathrm{HCl}(1 \mathrm{M})$ hydrolysis in the same reaction vessel, and the radiotracer was purified by semi-preparative HPLC. The identity of the radiotracer was confirmed using HPLC co-injection analysis. The radiochemical purity of $\left[{ }^{18} \mathrm{~F}\right]-\mathrm{THK} 5317$ was greater than 95\%, and its specific activities ranged from 90 to 123.5 GBq/ $\mu \mathrm{mol}$, which was corrected at the end of the synthesis.

\section{Positron Emission Tomography Data Preprocessing}

The MRI data were co-registered to the PET $\left[\left({ }^{18} \mathrm{~F}\right)\right.$-THK5317 and $\left.\left({ }^{11} \mathrm{C}\right)-\mathrm{PIB}\right]$ data and spatially normalized to a customized template in Montreal Neurological Institute (MNI) space, which was constructed from the MRI T1W images acquired in this study. The transformative deformation fields were applied to the corresponding PET data, and then isotropic $2 \mathrm{~mm}$ spatial resolution-normalized PET data in MNI space were generated. The mean value of the cerebellar gray matter region was used to normalize the intensity values of the PET images, voxel-byvoxel. A $6 \mathrm{~mm}$ FWHM Gaussian kernel was used to smooth the normalized PET data. The cut-off value for PIB-PET was 1.4 (Tanaka et al., 2020).

\section{Resting-State fMRI Data Preprocessing}

The resting-state fMRI data were preprocessed using SPM12 software $^{1}$. The first six volumes were discarded to allow for magnetization equilibrium and participants' adaption to the environment. The slice timing and rigid-body head movement during scans were corrected, so that all images were realigned to the first volume. The excessive motion was defined as a maximum displacement of $3 \mathrm{~mm}$ and a maximum angular motion of $3^{\circ}$ in

${ }^{1}$ http://www.fil.ion.ucl.ac.uk/spm any direction. The structural images were co-registered to the first volume of the corresponding functional images, and then segmented into gray matter, white matter, and cerebrospinal fluid. All fMRI images were normalized to the MNI space following motion correction using a diffeomorphic non-linear registration algorithm (DARTEL) (Ashburner, 2007) and resampled to a 2$\mathrm{mm}$ isotropic voxel. The normalized fMRI images were smoothed using a Gaussian kernel of 6 mm FWHM. The fMRI images were finally filtered with a temporal band-path of $0.01-0.1 \mathrm{~Hz}$, and white matter and CSF signals were regressed out.

\section{Statistics}

Data were analyzed using SPSS, version 23.0 (IBM Corp., Chicago, IL, United States). Demographic and clinical variables were assessed for normality of distribution using KolmogorovSmirnov tests. Variables exhibiting a normal distribution were compared using the Student's $t$-test. Gender was analyzed using a Chi-square test. A $p$-value of $<0.05$ was considered significant.

A voxel-wise two-sample $t$-test was used to compare the difference of the $\left[{ }^{18} \mathrm{~F}\right]-$ THK5317 cortical-to-cerebellum standardized uptake value ratio (SUVR) between the AD and $\mathrm{HC}$ groups. The false discovery rate (FDR) for multiple comparisons was utilized to control the expected proportion of false-positive results among the suprathreshold voxels with a $p<0.05$ and a cluster size larger than $800 \mathrm{~mm} 3$. Significant group differences $(p<0.05)$ were used as ROIs for rs-fMRI network construction.

Seven regions of interest (ROIs) were extracted from brain regions where tau deposition was significantly increased in the $\mathrm{AD}$ group compared to the $\mathrm{HC}$ group (details are presented in the "Results" section). An FC analysis was performed among the given ROIs, which was measured using Pearson's correlation coefficient. The correlation coefficient was derived between the mean time series of each pair of the seven ROIs, with each subject as the FC. A two-sample $t$-test was conducted to compare the FC difference between the two groups for each ROI pair. A significance level of an uncorrected $p<0.05$ was obtained for the FC comparisons.

\section{RESULTS}

\section{Demographic Characteristics}

Table 1 lists the clinical and neuropsychological data. No significant differences were observed in age, gender, and education level. All AD participants had positive results from the PIB-PET scans. Also, the AD group exhibited significantly lower scores than the HC group for the MMSE.

\section{Voxel-Based Morphometry Analysis of Tau Deposition}

The results of the voxel-based morphometry (VBM) analysis of the SUVR maps are shown in Table 2 and Figure 1. The AD group exhibited a significantly higher SUVR than the HC group in numerous cortical and subcortical areas, including the bilateral posterior cingulate cortex (PCC), ventromedial prefrontal cortex, temporal cortex, and parietal cortex (Table 2 and Figure 1). No 
TABLE 1 | Clinical and demographic characteristics of all subjects.

\begin{tabular}{|c|c|c|c|}
\hline & HCs & AD patients & $p$ value \\
\hline $\mathrm{N}$ & 19 & 26 & - \\
\hline Age $(Y)$ & $65.59 \pm 8.05$ & $70.68 \pm 12.21$ & 0.10 \\
\hline Gender (M/F) & $9 / 8$ & $17 / 12$ & 0.29 \\
\hline Education (Y) & $10.73 \pm 4.99$ & $11.23 \pm 3.48$ & 0.421 \\
\hline MMSE* & $29.33 \pm 0.18$ & $20.27 \pm 4.69$ & $<0.001$ \\
\hline
\end{tabular}

*MMSE scores for AD patients vs. HCs were significantly different by a two-sample t-test, $p<0.05$. Data are mean \pm SD or numbers of subjects.

TABLE 2 | Significant clusters of higher tau accumulation in Alzheimer's disease.

\begin{tabular}{|c|c|c|c|c|c|c|c|}
\hline \multirow[t]{2}{*}{ No } & \multirow[t]{2}{*}{ Cluster voxels } & \multirow[t]{2}{*}{ Lateralization } & \multirow[t]{2}{*}{ Regions } & \multirow[t]{2}{*}{$T$} & \multicolumn{3}{|c|}{ MNI coordinate } \\
\hline & & & & & $\mathbf{x}$ & $\mathbf{Y}$ & $\mathbf{Z}$ \\
\hline 1 & 8,795 & Left & $\begin{array}{l}\text { Middle and inferior temporal cortex, Middle and } \\
\text { inferior occipital cortex, Angular cortex, Precuneus } \\
\text { cortex, Parahippocampal cortex }\end{array}$ & -7.827 & -22 & -74 & 44 \\
\hline 2 & 10,995 & Right & $\begin{array}{l}\text { Temporal cortex, Middle and inferior occipital } \\
\text { cortex, Angular cortex, Precuneus cortex, } \\
\text { Parahippocampal cortex }\end{array}$ & -7.821 & 52 & -60 & -18 \\
\hline 3 & 4,791 & Left & Dorsal lateral prefrontal cortex & -7.015 & -36 & 32 & 34 \\
\hline 4 & 4,986 & Right & Dorsal lateral prefrontal cortex & -8.390 & 22 & 16 & 60 \\
\hline 5 & 105 & Right & Parahippocampus & -4.661 & 20 & -14 & -16 \\
\hline 6 & 479 & Left & Caudate & -6.383 & 0 & 18 & -4 \\
\hline 7 & 4,401 & Bilateral & Middle and posterior cingulate cortex & -8.087 & 8 & -34 & 44 \\
\hline
\end{tabular}

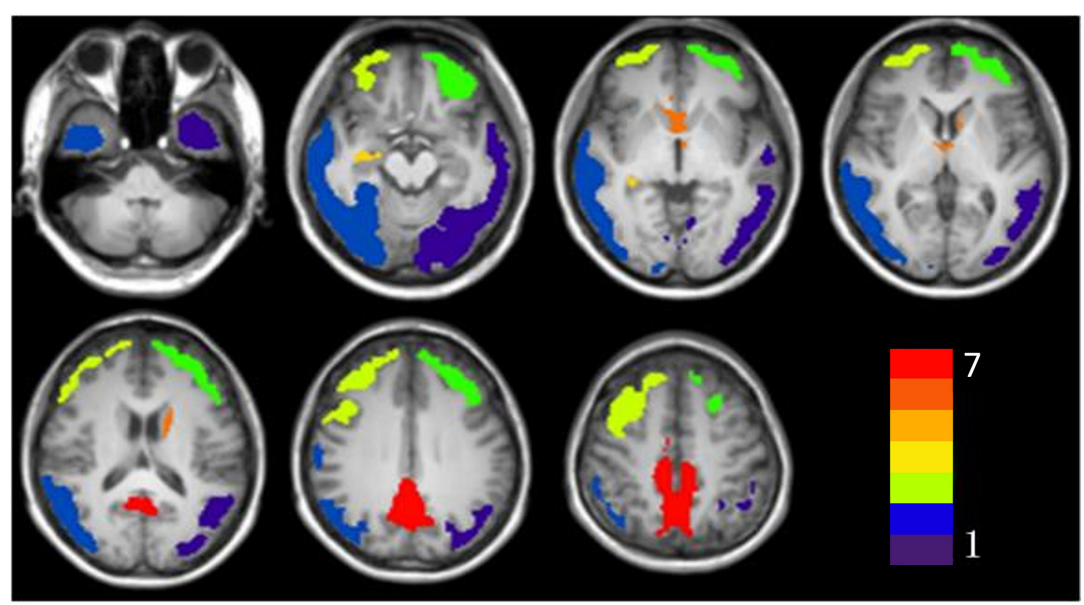

FIGURE 1 | The significant clusters of tau accumulation in Alzheimer's disease patients. The color bar indicates the different clusters (Cluster 1 to Cluster 7 ).

region was observed that had a significantly lower SUVR in the AD group compared to the HC group.

\section{Functional Connectivity Analysis in Regions of Tau Deposition}

Seven clusters were extracted from the VBM results and used as the ROIs in the FC analysis. The FC between each two ROIs was calculated as the correlation between their time series for all subjects. Compared with the HC group, the AD group showed a significantly decreased connectivity pattern between Clusters 1 and 2 (Table 3). We also found a negative correlation between the FC and SUVR between Clusters 1 and 2 (Figure 2).

\section{Exploratory Analysis: Clinical and Radiological Characteristics of the Functional Connectivity-Positive and Functional Connectivity-Negative Alzheimer's Disease Subgroups}

In the exploratory analysis, we observed that 12 subjects in the $\mathrm{AD}$ group showed a negative FC between Clusters 1 and 2. This 
TABLE 3 | Group differences of the FC for each ROI pair between the AD and HC groups.

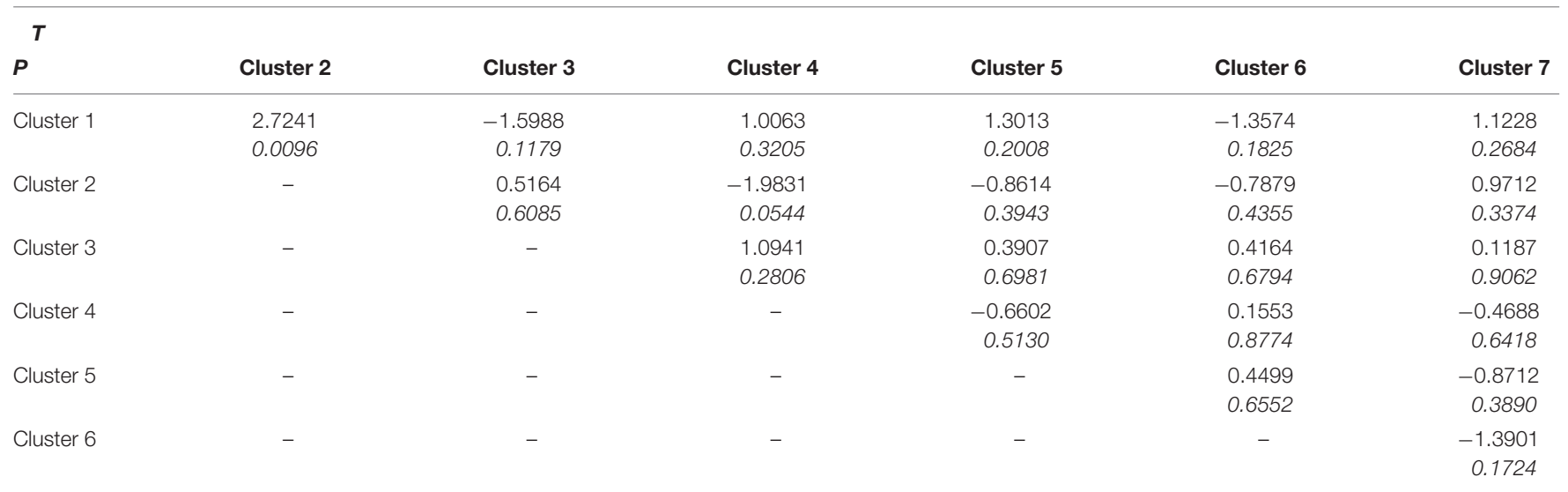

In each cell, the upper number is the T value, and the lower number is the $p$-value for each ROI pair.

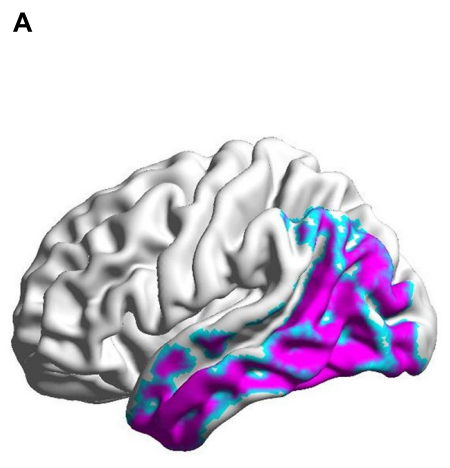

\section{Cluster 1}

B

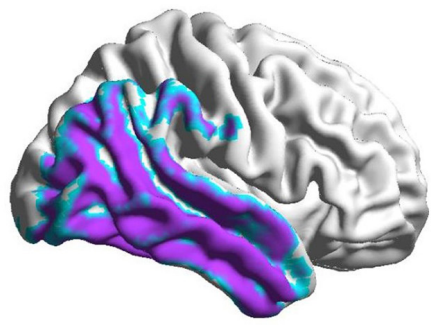

Cluster 2
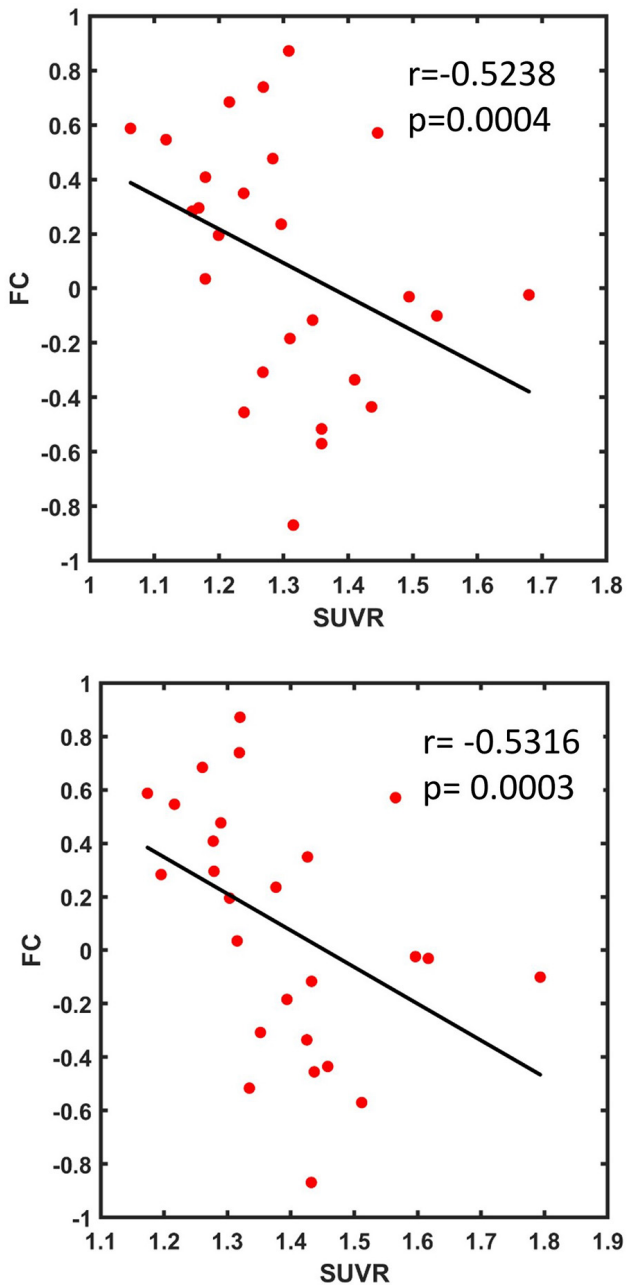

FIGURE 2 | Scatter plot of the functional connectivity and standardized uptake value ratio (SUVR) in Cluster 1 (A) and Cluster 2 (B) in Alzheimer's disease patients.

result indicated that the correlation of paired BOLD signals was negatively correlated, while the other 14 subjects in the AD group showed a positive FC between Clusters 1 and 2. However, all subjects in the HC group exhibited a positive FC between Clusters 1 and 2, which indicated that the correlation was positive or synergic (Figure 3 and Supplementary Material 1). 

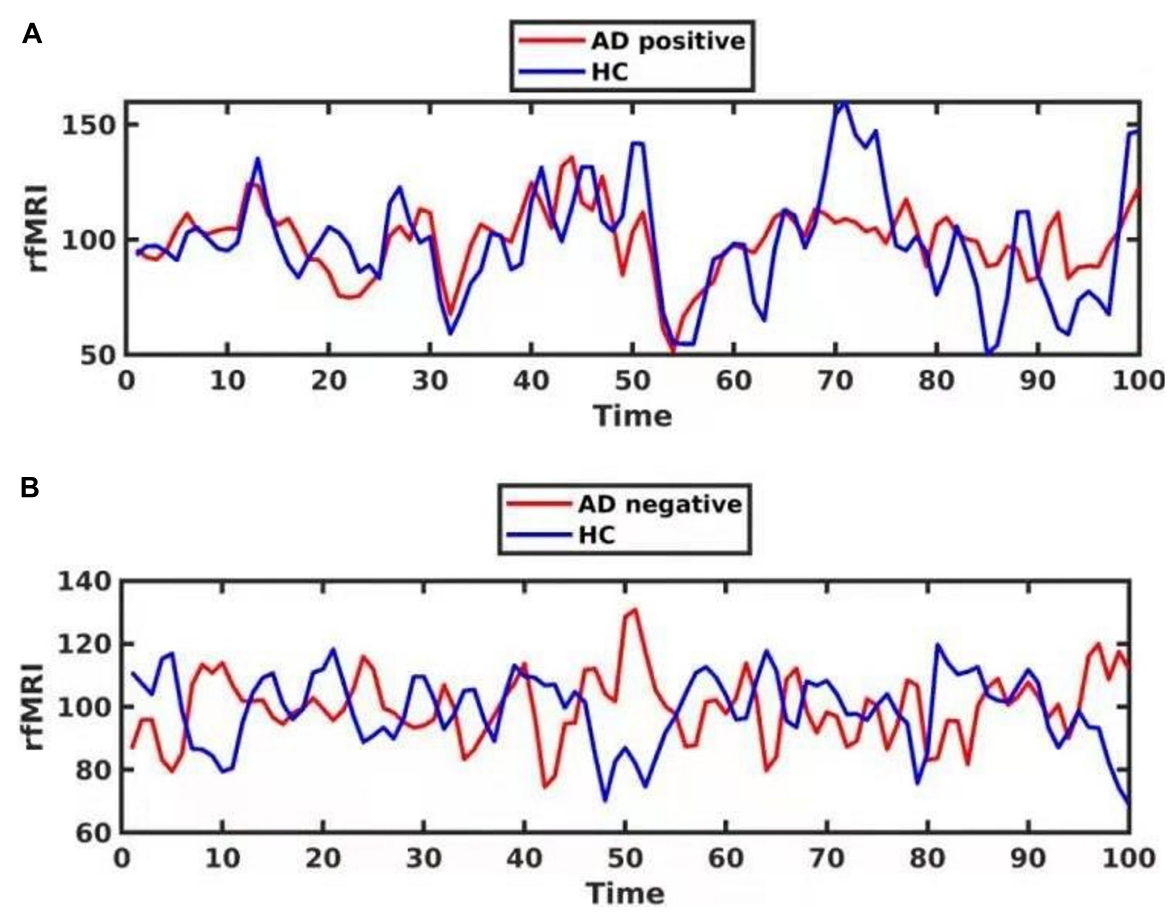

FIGURE 3 | The phase delay of the functional BOLD series for one Alzheimer's disease (AD) patient with positive functional connectivity (FC) [(A), red] and another AD patient with negative FC [(B), red]. The FC is between Cluster 1 and Cluster 2. The time series for one healthy control is shown in blue.

Based on the FC between Clusters 1 and 2, we divided the $\mathrm{AD}$ group into FC-positive and FC-negative subgroups. Table 4 illustrates the clinical and radiological characteristics of these two $\mathrm{AD}$ subgroups. When compared to the FC-positive patients, the FC-negative patients were younger and exhibited lower MMSE scores. No significant difference of GMV in Clusters 1 and 2 was detected in the FC-negative and FC-positive subgroups. However, the FC-negative group had greater $\left[{ }^{18} \mathrm{~F}\right]$-THK5317 binding in Clusters 1 and 2.

\section{DISCUSSION}

The main result of this cross-sectional study was the presence of higher tau deposition in the $\mathrm{AD}$ group in the bilateral inferior lateral temporal lobe, dorsal prefrontal cortex, precuneus, posterior cingulate cortex, hippocampus, and occipital lobe. FC analysis revealed decreased FC in regions with higher $\left[{ }^{18} \mathrm{~F}\right]-$ THK5317 signals. The FC strength was negatively correlated with the regional SUVR in patients with AD. Exploratory analysis revealed that patients with a positive $\mathrm{FC}$ were older and exhibited better cognitive performance than patients with a negative FC. In addition, tau accumulation was higher in patients with a negative FC than those with a positive FC. Taken together, these findings suggested that tau accumulation impacted the function and clinical performance of $\mathrm{AD}$ patients.

Pathological proteins, including tau, either directly or indirectly, interfere with cerebral function and morphology in $\mathrm{AD}$. Previous studies have demonstrated disruptions of large-scale brain networks in $\mathrm{AD}$, including the default-mode network (DMN) and other networks. However, little is known about alterations in FC patterns associated with high tau deposition. Consistent with previous studies, we demonstrated that regions of tau accumulation were located in important hubs of the posterior DMN, including the precuneus and angular cortex (Franzmeier et al., 2019). With respect to cognitive function, episodic memory is the most vulnerable cognitive subdomain in early $\mathrm{AD}$ and relies on the interaction between the DMN and the medial temporal lobe (Ward et al., 2015). Executive function also declined in concert with memory in the early stage of $\mathrm{AD}$, implicating an interplay between the DMN and other relevant networks (La Corte et al., 2016). In $\mathrm{AD}$, where tau accumulation predominates, the affected nodes become weakly connected, which reduces the local efficiency of information transfer.

Negative FC refers to a negative Pearson correlation coefficient for the spontaneous BOLD signal in two brain regions, indicating a negative correlation for the two regions. The origin and interpretation of a negative FC have been debated. Some studies have reported that a negative FC was an artifact induced by the global signal regression and excluded results that included a negative FC to avoid uncertainty (Weissenbacher et al., 2009). However, other studies found that a negative FC could exist without a global signal regression, and the characteristics of a negatively correlated network were not related to the global signal removal (Chang and Glover, 2009; Fox et al., 2009). Additional studies have revealed that a negative FC was associated with predominantly long-range 
TABLE 4 | Demographic and radiological characteristics of the FC-positive and FC-negative groups in AD patients.

\begin{tabular}{|c|c|c|c|c|c|}
\hline & & FC-negative AD & FC-positive AD & Z score & $p$ value \\
\hline$n$ & & 12 & 14 & & \\
\hline Age $^{\star}$ & & $66.08 \pm 9.79$ & $74.71 \pm 12.32$ & -2.04 & 0.044 \\
\hline Gender (M/F) & & $4: 8$ & $7: 7$ & & 0.46 \\
\hline MMSE* $^{\star}$ & & $17.00 \pm 5.31$ & $22.71 \pm 2.84$ & -2.51 & 0.012 \\
\hline \multirow[t]{2}{*}{ Cluster 1} & GMV & $5,687.31 \pm 1,050.03$ & $6,120.13 \pm 920.18$ & -0.93 & 0.374 \\
\hline & SUVR* & $1.40 \pm 0.13$ & $1.22 \pm 0.95$ & -3.45 & 0.0005 \\
\hline \multirow[t]{2}{*}{ Cluster 2} & GMV & $6,981.53 \pm 1,250.72$ & $7,570.47 \pm 1,135.87$ & -1.18 & 0.237 \\
\hline & SUVR* & $1.48 \pm 0.13$ & $1.31 \pm 0.10$ & -3.55 & 0.0009 \\
\hline
\end{tabular}

*Age, MMSE scores, and SUVR in cluster 1 and cluster 2 for FC-negative AD vs. FC-positive AD patients were significantly different based on a two-sample t-test, $p<0.05$. Data are means $\pm S D$ or numbers of subjects.

connections, which provides a possible explanation for the underlying neurobiological mechanism. Moreover, Chen et al. (2011) suggested that a negative FC might induce a phase delay in the synchronous signals along the shortest path in the brain functional networks. Because the mechanisms of negative FC still are not well understood (Chen et al., 2011), we compared the cognitive performance between the FCpositive and FC-negative $\mathrm{AD}$ subgroups. We observed that $\mathrm{AD}$ patients with a positive $\mathrm{FC}$ in the tau accumulation region performed better in the cognitive test. Also, the SUVR for $\left[{ }^{18} \mathrm{~F}\right]$-THK5317 was higher in patients with a negative FC. It is worth mentioning that a negative FC was only found in the $\mathrm{AD}$ group. These findings support the biological mechanism of negative FC.

In this study, compared with the negative FC subgroup, the positive FC subgroup might be protective to compensate for the neuron injury caused by tau deposition and allows the cognitive performance to be maintained. This mechanism might lead to a period of hyperactivity until the neuronal loss overcomes the compensatory mechanism. However, whether the reserve capacity is related to the cognitive benefit in the presence of severe tau accumulation is controversial. Previous pathological studies suggested that the reserve capacity is related to decreased cognitive impairment in the presence of $A \beta$ pathology but not tau pathology. However, a recent study using tau-PET found that a higher intelligence quotient was associated with an attenuated association between tau accumulation and cognitive decline (Halawa et al., 2019). Additional exploration of the cognitive reserve capacity will help identify individuals with a higher tolerance of tau pathologic burden in future studies, and help in the early diagnosis and intervention for $\mathrm{AD}$ patients.

If the network efficiency relates to the cognitive and clinical performance, then the FC alteration would predict the neurodegenerative process, particularly for the tau accumulation in hub regions. A negative FC might indicate a connectivity disruption in the temporal lobe and parietal cortex, which leads to an advanced stage of AD.

$\left[{ }^{18} \mathrm{~F}\right]$-THK5117 has shown a high affinity for and selective binding to tau pathology (Harada et al., 2015; Lemoine et al., 2015). Its $S$-form enantiomer [also known as $\left({ }^{18} \mathrm{~F}\right)$-THK5317] has exhibited favorable pharmacokinetics (Jonasson et al., 2016). It was reported that, except for tau deposition in the neocortex, the monoamine oxidase- $\mathrm{B}$ (MAO-B) in the entire brain is correlated with retention of $\left[{ }^{18} \mathrm{~F}\right]-\mathrm{THK} 5317$ (Harada et al., 2018). However, MAO-B is primarily localized in the inner mitochondrial membrane of astrocytes and linked to the presence of astrogliosis with the accumulation of misfolded proteins. In addition to tau deposition, the activation of microglia and astrogliosis also contributes to the development of AD (Leyns and Holtzman, 2017). Therefore, $\left[{ }^{18} \mathrm{~F}\right]-$ THK5317 retention in the AD neocortex is expected to indicate the distribution of tau pathology and reflect the presence of reactive astrocytes in vivo.

There are several limitations to this study. First, although all $\mathrm{AD}$ participants exhibited positive PIB-PET results, we did not include the influence of $A \beta$. Schultz et al. (2017) found that tau and $\mathrm{A} \beta$ both affect $\mathrm{FC}$, and tau-FC associations were stronger and increased with A $\beta$ levels (Schultz et al., 2017; Adams et al., 2019; Franzmeier et al., 2019). Second, in addition to tau, $\left[{ }^{18} \mathrm{~F}\right]$-THK5317 retention has been reported to reflect reactive astrocytes (Shigemoto et al., 2018). Third, only the MMSE scores were used to evaluate the cognitive level of $\mathrm{AD}$ patients. More subdomain evaluations, such as memory, executive function, and others, are needed to assess the cognition of $\mathrm{AD}$ patients more accurately. Finally, the cross-sectional nature and small sample size limited our interpretation of causality. Additional longitudinal studies with larger sample sizes are needed to investigate whether tau deposits accurately predict atrophy and decreases in FC.

\section{CONCLUSION}

Therefore, the cortical regions, including the bilateral inferior lateral temporal lobe, dorsal prefrontal cortex, precuneus, posterior cingulate cortex, hippocampus, and occipital lobe, showed significantly higher $\left[{ }^{18} \mathrm{~F}\right]$-THK5317 accumulation in patients with AD. Decreased FC in regions with higher SUVR was observed in $\mathrm{AD}$ patients, and the FC strength negatively correlated with regional SUVR. Patients with a positive FC exhibited older ages, better cognitive performances, and a lower SUVR than patients with a negative FC. The current results indicated that there was an impact of tau deposition on FC at the individual level in AD patients. Furthermore, our findings suggested that the combination of tau-PET and rs-fMRI might be useful to predict the progression of $\mathrm{AD}$. 


\section{DATA AVAILABILITY STATEMENT}

The original contributions presented in the study are included in the article/Supplementary Material, further inquiries can be directed to the corresponding author/s.

\section{ETHICS STATEMENT}

The studies involving human participants were reviewed and approved by the Chinese PLA General Hospital. The patients/participants provided their written informed consent to participate in this study.

\section{AUTHOR CONTRIBUTIONS}

LF contributed to the research concept and design, data analysis and interpretation, drafting of the manuscript, critical revision of the article, and final approval of the article. ZZ and LL contributed to data analysis and data interpretation. JZ and $\mathrm{XZ}$ contributed to radiosynthesis and data analysis. HX and MZ contributed to data collection and interpretation. RW contributed to the research concept and design. All authors contributed to the article and approved the submitted version.

\section{REFERENCES}

Adams, J. N., Maass, A., Harrison, T. M., Baker, S. L., and Jagust, W. J. (2019). Cortical tau deposition follows patterns of entorhinal functional connectivity in aging. Elife 8:e49132. doi: 10.7554/eLife.49132.026

Ashburner, J. (2007). A fast diffeomorphic image registration algorithm. Neuroimage 38, 95-113. doi: 10.1016/j.neuroimage.2007.07.007

Busche, M. A., Wegmann, S., Dujardin, S., Commins, C., Schiantarelli, J., Klickstein, N., et al. (2019). Tau impairs neural circuits, dominating amyloidbeta effects, in Alzheimer models in vivo. Nat. Neurosci. 22, 57-64. doi: 10. 1038/s41593-018-0289-8

Chang, C., and Glover, G. H. (2009). Effects of model-based physiological noise correction on default mode network anti-correlations and correlations. Neuroimage 47, 1448-1459. doi: 10.1016/j.neuroimage.2009.05.012

Chen, G., Chen, G., Xie, C., and Li, S. J. (2011). Negative functional connectivity and its dependence on the shortest path length of positive network in the resting-state human brain. Brain Connect. 1, 195-206. doi: 10.1089/brain.2011. 0025

Cope, T. E., Rittman, T., Borchert, R. J., Jones, P. S., Vatansever, D., Allinson, K., et al. (2018). Tau burden and the functional connectome in Alzheimer's disease and progressive supranuclear palsy. Brain 141, 550-567. doi: 10.1093/brain/ awx347

Fox, M. D., Zhang, D., Snyder, A. Z., and Raichle, M. E. (2009). The global signal and observed anticorrelated resting state brain networks. J. Neurophysiol. 101, 3270-3283. doi: 10.1152/jn.90777.2008

Franzmeier, N., Neitzel, J., Rubinski, A., Smith, R., Strandberg, O., Ossenkoppele, R., et al. (2020). Functional brain architecture is associated with the rate of tau accumulation in Alzheimer's disease. Nat. Commun. 11:347. doi: 10.1038/ s41467-019-14159-1

Franzmeier, N., Rubinski, A., Neitzel, J., Kim, Y., Damm, A., Na, D. L., et al. (2019). Functional connectivity associated with tau levels in ageing. Alzheimer's, and small vessel disease. Brain 142, 1093-1107. doi: 10.1093/brain/awz026

Halawa, O. A., Gatchel, J. R., Amariglio, R. E., Rentz, D. M., Sperling, R. A., Johnson, K. A., et al. (2019). Inferior and medial temporal tau and cortical amyloid are associated with daily functional impairment in Alzheimer's disease. Alzheimers Res. Ther. 11:14. doi: 10.1186/s13195-019-0471-6

\section{FUNDING}

This study was financially sponsored by the National Key Research and Development Program of China under grant (No. 2016YFC0103804), Beijing Municipal Natural Science Foundation (No. 7192192) and National Natural Science Foundation of China (Nos. 82071963 and 11975249).

\section{ACKNOWLEDGMENTS}

We would like to thank Dayi Yin and Jiajin Liu for technical support and PET/MRI data acquisition, as well as Jian Liu and Yungang Li for their help with the PET radiochemistry. We would also like to thank EditSprings (https://www.editsprings. $\mathrm{com} /$ ) for their expert linguistic services.

\section{SUPPLEMENTARY MATERIAL}

The Supplementary Material for this article can be found online at: https://www.frontiersin.org/articles/10.3389/fnagi. 2021.758053/full\#supplementary-material

Harada, R., Ishiki, A., Kai, H., Sato, N., Furukawa, K., Furumoto, S., et al. (2018). Correlations of (18)F-THK5351 PET with postmortem burden of tau and astrogliosis in Alzheimer disease. J. Nucl. Med. 59, 671-674. doi: 10.2967/ jnumed.117.197426

Harada, R., Okamura, N., Furumoto, S., Furukawa, K., Ishiki, A., Tomita, N., et al. (2015). [(18)F]THK-5117 PET for assessing neurofibrillary pathology in Alzheimer's disease. Eur. J. Nucl. Med. Mol. Imaging 42, 1052-1061. doi: 10.1007/s00259-015-3035-4

Jack, C. R. Jr., Bennett, D. A., Blennow, K., Carrillo, M. C., Dunn, B., et al. (2018). NIA-AA research framework: toward a biological definition of Alzheimer's disease. Alzheimers Dement. 14, 535-562. doi: 10.1016/j.jalz.2018. 02.018

Jonasson, M., Wall, A., Chiotis, K., Saint-Aubert, L., Wilking, H., Sprycha, M., et al. (2016). Tracer kinetic analysis of (S)- ${ }^{18} 8$ F-THK5117 as a PET tracer for assessing tau pathology. J. Nucl. Med. 57, 574-581. doi: 10.2967/jnumed.115. 158519

Kametani, F., and Hasegawa, M. (2018). Reconsideration of amyloid hypothesis and tau hypothesis in Alzheimer's disease. Front. Neurosci. 12:25. doi: 10.3389/ fnins.2018.00025

La Corte, V., Sperduti, M., Malherbe, C., Vialatte, F., Lion, S., Gallarda, T., et al. (2016). Cognitive decline and reorganization of functional connectivity in healthy aging: the pivotal role of the salience network in the prediction of age and cognitive performances. Front. Aging Neurosci. 8:204. doi: 10.3389/fnagi. 2016.00204

Lemoine, L., Saint-Aubert, L., Marutle, A., Antoni, G., Eriksson, J. P., Ghetti, B., et al. (2015). Visualization of regional tau deposits using (3)H-THK5117 in Alzheimer brain tissue. Acta Neuropathol. Commun. 3:40. doi: 10.1186/s40478015-0220-4

Leyns, C. E. G., and Holtzman, D. M. (2017). Glial contributions to neurodegeneration in tauopathies. Mol. Neurodegener. 12:50. doi: 10.1186/ s13024-017-0192-x

McKhann, G. M., Knopman, D. S., Chertkow, H., Hyman, B. T., Jack, C. R., et al. (2011). The diagnosis of dementia due to Alzheimer's disease: recommendations from the national institute on Aging-Alzheimer's association workgroups on diagnostic guidelines for Alzheimer's disease. Alzheimers Dement. 7, 263-269. doi: 10.1016/j.jalz.2011.03.005 
McNamee, R. L., Yee, S. H., Price, J. C., Klunk, W. E., Rosario, B., Weissfeld, L., et al. (2009). Consideration of optimal time window for pittsburgh compound B PET summed uptake measurements. J. Nucl. Med. 50, 348-355. doi: 10.2967/ jnumed.108.057612

Morris, J. C. (1993). The clinical dementia rating (CDR): current version and scoring rules. Neurology 43, 2412-2414. doi: 10.1212/WNL.43.11.2412-a

Philippe, C., Haeusler, D., Mitterhauser, M., Ungersboeck, J., Viernstein, H., Dudczak, R., et al. (2011). Optimization of the radiosynthesis of the Alzheimer tracer 2-(4-N-[11C]methylaminophenyl)-6-hydroxybenzothiazole ([11C]PIB). Appl. Radiat. Isot. 69, 1212-1217. doi: 10.1016/j.apradiso.2011.04.010

Scheltens, P., Leys, D., Barkhof, F., Huglo, D., Weinstein, H. C., Vermersch, P., et al. (1992). Atrophy of medial temporal lobes on MRI in "probable" Alzheimer's disease and normal ageing: diagnostic value and neuropsychological correlates. J. Neurol. Neurosurg. Psychiatry 55, 967-972. doi: 10.1136/jnnp.55. 10.967

Schultz, A. P., Chhatwal, J. P., Hedden, T., Mormino, E. C., Hanseeuw, B. J., Sepulcre, J., et al. (2017). Phases of hyperconnectivity and hypoconnectivity in the default mode and salience networks track with amyloid and tau in clinically normal individuals. J. Neurosci. 37, 4323-4331. doi: 10.1523/JNEUROSCI. 3263-16.2017

Schwarz, A. J., Yu, P., Miller, B. B., Shcherbinin, S., Dickson, J., Navitsky, M., et al. (2016). Regional profiles of the candidate tau PET ligand 18F-AV-1451 recapitulate key features of Braak histopathological stages. Brain 139(Pt 5), 1539-1550. doi: 10.1093/brain/aww023

Shigemoto, Y., Sone, D., Imabayashi, E., Maikusa, N., Okamura, N., Furumoto, S., et al. (2018). Dissociation of tau deposits and brain atrophy in early Alzheimer's disease: a combined positron emission tomography/magnetic resonance imaging study. Front. Aging Neurosci. 10:223. doi: 10.3389/fnagi. 2018.00223

Tanaka, T., Stephenson, M. C., Nai, Y. H., Khor, D., Saridin, F. N., Hilal, S., et al. (2020). Improved quantification of amyloid burden and associated biomarker cut-off points: results from the first amyloid Singaporean cohort with overlapping cerebrovascular disease. Eur. J. Nucl. Med. Mol. Imaging 47, 319-331. doi: 10.1007/s00259-019-04642-8

Ward, A. M., Mormino, E. C., Huijbers, W., Schultz, A. P., Hedden, T., and Sperling, R. A. (2015). Relationships between default-mode network connectivity, medial temporal lobe structure, and age-related memory deficits. Neurobiol. Aging 36, 265-272. doi: 10.1016/j.neurobiolaging.2014.06.028

Weissenbacher, A., Kasess, C., Gerstl, F., Lanzenberger, R., Moser, E., and Windischberger, C. (2009). Correlations and anticorrelations in resting-state functional connectivity MRI: a quantitative comparison of preprocessing strategies. Neuroimage 47, 1408-1416. doi: 10.1016/j.neuroimage.2009.05.005

Yokoi, T., Watanabe, H., Yamaguchi, H., Bagarinao, E., Masuda, M., and Imai, K. (2018). Involvement of the Precuneus/Posterior Cingulate Cortex is significant for the development of Alzheimer's disease: a PET (THK5351, PiB) and resting fMRI study. Front. Aging Neurosci. 10:304. doi: 10.3389/fnagi.2018.00304

Conflict of Interest: The authors declare that the research was conducted in the absence of any commercial or financial relationships that could be construed as a potential conflict of interest.

Publisher's Note: All claims expressed in this article are solely those of the authors and do not necessarily represent those of their affiliated organizations, or those of the publisher, the editors and the reviewers. Any product that may be evaluated in this article, or claim that may be made by its manufacturer, is not guaranteed or endorsed by the publisher.

Copyright (C) $2021 \mathrm{Fu}$, Zhou, Liu, Zhang, Xie, Zhang, Zhu and Wang. This is an open-access article distributed under the terms of the Creative Commons Attribution License (CC BY). The use, distribution or reproduction in other forums is permitted, provided the original author(s) and the copyright owner(s) are credited and that the original publication in this journal is cited, in accordance with accepted academic practice. No use, distribution or reproduction is permitted which does not comply with these terms. 